\title{
DISEÑO ESTÁTICO DE EDIFICIOS DE VARIOS PISOS SUSCEPTIBLES A TORSIÓN SÍSMICA
}

\author{
Jaime de la Colina Martínez, ${ }^{(1)}$
}

\begin{abstract}
RESUMEN
Se presentan los resultados de un trabajo analítico enfocado al estudio de edificios de cortante de varios pisos con desbalance torsional y elementos resistentes orientados en dos direcciones ortogonales. Se consideran modelos con asimetría en la distribución de masas y modelos con asimetría de rigideces. Los modelos se diseñan con siete grupos de recomendaciones asociadas al método estático de diseño por torsión, omitiendo los efectos relacionados con excentricidades accidentales. Se calculan las demandas de ductilidad de algunos elementos resistentes ante la acción de tres movimientos sísmicos bidireccionales. Se ofrecen recomendaciones para diseño por torsión.
\end{abstract}

\begin{abstract}
Results of an analytical work for the study of torsionally unbalanced multistory shear buildings are presented. Structural models include lateral resisting elements oriented along two orthogonal directions. Mass and stiffness eccentricities are considered separately. Seven groups of design recommendations associated with the static method for torsion are assessed. Accidental eccentricity is not considered. Displacement ductility demands of lateral resisting elements are computed for the models subjected to three bidirectional ground motions. Torsion design recommendations are given.
\end{abstract}

Artículo recibido el 20 de febrero del 2001 y aprobado para su publicación el 26 de abril de 2001. Se aceptarán comentarios y/o discusiones hasta cinco meses después de su publicación.

(1) Facultad de ingeniería, Universidad Autónoma del Estado de México jcolina@coatepec.uaemex.mx. 


\section{INTRODUCCIÓN}

La torsión sísmica en edificios es una forma de comportamiento estructural estudiada ampliamente en una gran cantidad de investigaciones, principalmente analíticas. Sin embargo, debido a la complejidad relativa del problema, la gran mayoría de las investigaciones han considerado un modelo de un piso para explicar el comportamiento de edificios de varios niveles.

Pocos son los estudios que abordan el problema de torsión usando modelos de varios entrepisos no lineales. Por ejemplo, Duan y Chandler [1] investigaron el efecto de los modos de vibrar superiores en la respuesta a torsión. También estudiaron varias recomendaciones de reglamentos de construcción y la utilidad de usar análisis modal en el proceso de diseño. Su estudio consideró excitación sísmica en una dirección y un modelo estructural con elementos resistentes a carga lateral (marcos) orientados también en una sola dirección. Se calcularon demandas de ductilidad del elemento del lado rígido del sistema. Algunos de sus resultados en modelos de 3 y 8 pisos indican que: a) en diseños basados en métodos estáticos, la aplicación de una fuerza concentrada en el último piso del edificio reduce significativamente la respuesta inelástica de las columnas de los pisos superiores en comparación con lo que se obtendría de un diseño basado en análisis modal o en distribuciones lineales simples del cortante y b) el análisis modal elástico no es efectivo para el diseño por torsión.

En un estudio posterior, Chandler y Duan [2] propusieron algunas recomendaciones para el diseño por torsión basadas en un análisis estático y en observaciones de un modelo de un piso, como sigue: a) una fórmula (Ecuaciones la y 1b) para calcular la excentricidad de diseño (primaria) en función de la excentricidad de entrepiso $e_{s} ; b$ ) un factor para la excentricidad de diseño (secundaria) basada en una reducción de sólo el 50\% del cortante para los elementos favorecidos por la torsión; y c) el uso de un factor (variable con la excentricidad) para amplificar las fuerzas de diseño del elemento del lado rígido. La aplicación de estas recomendaciones a sistemas "regularmente asimétricos" de 5 y 8 pisos, con periodos fundamentales de $0.5 \mathrm{~s}$ y $0.8 \mathrm{~s}$ respectivamente, llevó a demandas de ductilidad importantes en ciertos niveles para el elemento 1 (rígido) para excentricidades grandes. Esto último comprueba que las recomendaciones obtenidas de modelos de un piso no aplican directamente a modelos de varios pisos. Estudios presentados en la misma referencia en modelos de varios pisos sugieren el uso de una fuerza concentrada en el último piso $\left(\mathrm{F}_{\mathrm{t}}=10 \%\right.$ del cortante basal $)$ para reducir la respuesta inelástica de las columnas del lado rígido en los últimos entrepisos. Su estudio mostró que para modelos de ocho pisos, la inclusión de esta fuerza resulta en demandas de ductilidad de los elementos rígido y flexible muy cercanas a las correspondientes de sistemas torsionalmente balanceados para todos los entrepisos. Las ecuaciones que presentan Chandler y Duan [2] para calcular el factor de amplificación $\alpha$ son las siguientes:

$e_{D 1}=\alpha e_{s}$

donde

$\alpha=\left[2.6-3.6\left(e_{s} / b\right)\right] \geq 1.4$ 
En otro trabajo, Páez y Escobar [3] extendieron la recomendación de Tso y Wong [4] relacionada con el control de la rigidez torsional para controlar las demandas de ductilidad excesivas en edificios de varios pisos. Ellos concluyen que para lograr que la respuesta de las estructuras asimétricas permanezca dentro de límites adecuados de comportamiento, es necesario revisar los valores de la restricción a la torsión y la relación de rigideces que presentan los entrepisos. En su estudio, Páez y Escobar usaron modelos de varios niveles con elementos resistentes y acción sísmica orientados en una sola dirección.

De la Llera y Chopra [5] también estudiaron el comportamiento torsional de edificios de varios (cinco) pisos considerando un comportamiento elasto-plástico de los elementos resistentes a carga lateral. Los modelos empleados incluyeron elementos resistentes orientados en dos direcciones ortogonales y sujetos a las dos componentes del registro de El Centro (1940). La parte de su estudio relacionada con edificios de varios pisos se enfocó a investigar el efecto de: a) la resistencia de elementos orientados en dirección ortogonal (a la dirección principal del sismo), b) la asimetría de rigidez, c) la asimetría de resistencia, d) la distribución en planta de la resistencia, e) el número de elementos resistentes y f) el movimiento bidireccional del suelo. Sus principales resultados indican que: a) un incremento en la resistencia lateral de los elementos resistentes conduce a demandas de desplazamiento más uniformes de los elementos resistentes orientados en dirección de la componente principal del movimiento del terreno, b) la capacidad del sistema no se ve afectada por la excentricidad de rigidez del sistema, c) la asimetría de resistencia modifica el mecanismo de falla del sistema, d) los cambios en la distribución en planta de la resistencia conduce a cambios en la capacidad torsional del sistema, e) un incremento en el número de planos resistentes conduce a cambios pequeños de la respuesta y f) la componente ortogonal del movimiento sísmico implica una reducción de la capacidad torsional del sistema.

Duan y Chandler [6] también estudiaron una clase de edificios a base de marcos con discontinuidades con la altura y propusieron un procedimiento de diseño, similar al presentado en la referencia 2, para este tipo de edificios. El modelo usado incluye elementos resistentes y excitación sísmica en una sola dirección.

Del análisis de estos estudios también se obtienen las siguientes observaciones relacionadas con edificios de varios pisos:

a) Es claro que las recomendaciones de diseño, obtenidas en su mayoría del estudio de sistemas de un piso, deben evaluarse a la luz del conocimiento adquirido de sistemas de varios pisos.

b) El análisis modal (elástico lineal) no necesariamente conduce a diseños aceptables para estructuras torsionalmente desbalanceadas [1,6]. Esto sugiere comprobar si el método estático, el cual es más simple que el dinámico, puede conducir a niveles aceptables de las demandas de ductilidad de este tipo de estructuras.

c) La mayor parte de los estudios emplea elementos resistentes en una sola dirección y excitación sísmica en la misma dirección. Esto confía en cierta equivalencia con modelos que incluyen elementos en dos direcciones sujetos a excitación bidireccional. Sin embargo, esta equivalencia sólo ha sido estudiada para modelos de un piso $[7,8]$ por lo que su extensión a modelos de varios 
pisos es aún más cuestionable. Por esto, es recomendable el uso de modelos con elementos resistentes y acción sísmica en ambas direcciones.

En el presente trabajo se estudian recomendaciones para diseño de sistemas torsionalmente desbalanceados (TD) con apoyo en el método estático. Básicamente éstas son similares a las propuestas por Chandler y Duan [2] pero no se incluye el factor de resistencia que se aplica al elemento del lado rígido ni la localización de los centros de resistencia [6]. A diferencia de los modelos empleados en las Refs. 2 y 6, aquí se consideran modelos con elementos resistentes (marcos) orientados en ambas direcciones y sujetos a excitación sísmica actuando en ambas direcciones. Los edificios consisten de modelos de 5 pisos con excentricidades ocasionadas por asimetría en la distribución de la masa o rigidez, como se explica adelante.

\section{MODELOS ESTRUCTURALES}

Se investigan sistemas de 5 pisos con dimensiones en planta de $5.0 \times 10.0 \mathrm{~m}(\mathrm{a} \times \mathrm{b})$ para todos los niveles y con 6 elementos resistentes ( 3 en cada dirección) en cada uno de los entrepisos como se muestra en la Figura 1. En todos los casos, la rigidez del elemento 2 (central) es igual a la del 3 (derecho). Los elementos resistentes se idealizan como elementos bilineales resistentes a flexión con rigidez después de la fluencia igual a $10 \%$ de la inicial. En todos los casos y niveles, la losa se supone con movimiento de cuerpo rígido en su plano. Para los modelos con masa excéntrica, el centro de masa se desplaza hacia la derecha como se ilustra en la misma figura. En todos los casos, las rigideces de los elementos 4,5 y 6 son iguales.

Por lo que se refiere a las variaciones de masa y rigidez con la altura, se consideran siete modelos, como se muestra en la Figura 2 donde sólo se identifican los marcos 1 y 3 por claridad:

Modelo 1: Caso simétrico de referencia con masas alineadas en la parte media y sin excentricidad de rigidez en todos los entrepisos.

$>$ Modelo 2: Masa excéntrica del último entrepiso $\left(\mathrm{e}_{5}=0.25 \mathrm{~b}\right)$.

$>$ Modelo 3: Masas con excentricidad creciente con la altura tal que $\mathrm{e}_{5}=0.25 \mathrm{~b}$.

$>$ Modelo 4: Todas las masas excéntricas $\left(\mathrm{e}_{\mathrm{j}}=0.25 \mathrm{~b}\right)$.

$>$ Modelo 5: Masas alineadas al centro pero con los elementos 1 con el doble de rigidez de los elementos 2 y 3 , i.e., $\mathrm{k}_{1}=2 \mathrm{k}_{2}=2 \mathrm{k}_{3}$.

$>$ Modelo 6: Masas alineadas al centro pero con todos los elementos 1 con el cuádruple de rigidez de los elementos 2 y 3 , i.e., $\mathrm{k}_{1}=4 \mathrm{k}_{2}=4 \mathrm{k}_{3}$.

> Modelo 7: Masas alineadas al centro pero con los elementos 1 con valores de la rigidez que crecen de arriba a abajo.

Para todos los casos el valor de la masa es igual en cada uno de los cinco niveles (un quinto de la masa total del edificio M). Las masas se seleccionaron para obtener un periodo natural de vibración del Modelo 1 aproximadamente igual a $\mathrm{T}=0.5 \mathrm{~s}$. calculado de acuerdo con la expresión simplificada del UBC-94 [9]. El valor calculado con el cociente de Rayleigh resultó igual a 0.49 s. Este periodo se seleccionó para generar resultados que puedan ser comparables con los de otros estudios similares [1, $2,5]$. También se seleccionó para que el periodo sea congruente con el número de pisos del modelo de 
cinco pisos y para que el periodo esté cerca de la parte alta de los espectros de respuesta de los registros empleados. La matriz de amortiguamiento del sistema (c) se propuso proporcional a la matriz de masa (m) con un valor de $5 \%$ del crítico correspondiente al primer modo de vibrar de la parte lineal (inicial), i.e., $\mathbf{c}=\mathrm{a}_{0} \mathbf{m}$ siendo $\mathrm{a}_{0}$ un escalar. Esta forma de amortiguamiento se seleccionó para usar solamente la estimación del primer modo de vibrar. De esta manera se obtienen niveles de amortiguamiento menores del valor seleccionado (5\% en este caso) para los modos superiores [13].

Estos modelos no pretenden ajustarse a casos reales. Sólo busca estudiar las demandas de ductilidad para distintas excentricidades de todos los entrepisos ocasionada por distintas configuraciones de masa y rigidez con la altura. Se pretende cubrir un grupo de edificios con excentricidad ocasionada por asimetría de masa y otro grupo con asimetría de rigideces. Los modelos propuestos permiten obtener una variedad amplia de excentricidades de entrepiso, como se muestra en la Tabla 1.

Tabla 1. Excentricidades de piso normalizadas $\mathrm{e}_{\mathrm{s}} / \mathrm{b}$.

\begin{tabular}{cccccccc}
\hline Entrepiso & Modelo 1 & Modelo 2 & Modelo 3 & Modelo 4 & Modelo 5 & Modelo 6 & Modelo 7 \\
\hline 5 & 0.0 & 0.250 & 0.250 & 0.250 & 0.125 & 0.250 & 0.000 \\
4 & 0.0 & 0.139 & 0.227 & 0.250 & 0.125 & 0.250 & 0.100 \\
3 & 0.0 & 0.104 & 0.208 & 0.250 & 0.125 & 0.250 & 0.167 \\
2 & 0.0 & 0.089 & 0.193 & 0.250 & 0.125 & 0.250 & 0.214 \\
1 & 0.0 & 0.083 & 0.183 & 0.250 & 0.125 & 0.250 & 0.250 \\
\hline
\end{tabular}

\section{PROCEDIMIENTOS DE DISEÑO CONSIDERADOS}

Definida la rigidez de los elementos resistentes, el diseño de éstos se reduce al cálculo de su resistencia de fluencia para los diferentes entrepisos. Estos valores se obtienen con ayuda de las dos excentricidades de diseño que se emplean en el método estático de diseño:

excentricidad primaria $=\mathrm{e}_{\mathrm{d} 1}=\alpha \mathrm{e}_{\mathrm{s}}+\beta \mathrm{b}$

excentricidad secundaria $=\mathrm{e}_{\mathrm{d} 2}=\delta \mathrm{e}_{\mathrm{s}}-\beta \mathrm{b}$

Como se sabe, $\alpha$ es un factor que amplifica la excentricidad calculada $e_{s}$. En este trabajo, $\mathrm{e}_{\mathrm{s}} \mathrm{se}$ obtiene tomando en cuenta la posición del cortante por arriba del entrepiso j que se considera [10]. Por su parte, $\delta$ es un factor que descuenta el cortante ocasionado por torsión que favorece a algunos elementos resistentes a carga lateral. En todos los procedimientos de diseño que se consideran en este estudio, se desprecia la excentricidad accidental $\mathrm{e}_{\mathrm{a}}=\beta \mathrm{b}$ ya que no se incluyen en la modelación los efectos relacionados con ella. Por ejemplo, no se considera en la modelación la incertidumbre de la posición de los centros de masas ni la de los valores de las rigideces de los elementos resistentes. 
Las recomendaciones de diseño estudiadas en este trabajo se han agrupado en siete procedimientos y en ellos se varían los valores de $\alpha$ y $\delta$, así como los valores de la fuerza a aplicar en el último piso $\left(\mathrm{F}_{\mathrm{t}}\right)$. Excepto por el valor de $\mathrm{F}_{\mathrm{t}}$, la distribución vertical de fuerzas sísmicas se considera lineal en todos los casos. Cada uno de los procedimientos se identifica con una letra minúscula, como se muestra en la Tabla 2. Es importante destacar que $\delta=1.0$, usado por las Normas Técnicas Complementarias para Diseño por Sismo [11], ha mostrado [12] que $\delta=1.0$ conduce a demandas de ductilidad excesivas; por lo que no se considera dicho valor en la Tabla 2.

Es importante destacar que la aplicación de los procedimientos de diseño aquí estudiados conduce a capacidades variables con la altura para los distintos entrepisos. En la práctica, sin embargo, esto no sucede y generalmente se asignan capacidades iguales a algunos entrepisos consecutivos. Esto último seguramente conduce a demandas de ductilidad menores de las aquí presentadas para algunos entrepisos.

Tabla 2. Procedimientos de diseño considerados en este estudio

\begin{tabular}{cccl}
\hline Procedimiento & $\alpha$ & $\delta$ & Fuerza en el último piso, $\mathrm{F}_{\mathrm{t}}$ \\
\hline $\boldsymbol{A}$ & 1.0 & 0.0 & $\mathrm{~F}_{\mathrm{t}}$ según UBC \\
$\boldsymbol{B}$ & 1.5 & 0.0 & $\mathrm{~F}_{\mathrm{t}}$ según UBC \\
$\boldsymbol{C}$ & 1.0 & 0.5 & $\mathrm{~F}_{\mathrm{t}}$ según UBC \\
$\boldsymbol{D}$ & 1.5 & 0.5 & $\mathrm{~F}_{\mathrm{t}}$ según UBC \\
$\boldsymbol{E}$ & según Ec. 1 & 0.5 & $\mathrm{~F}_{\mathrm{t}}=10 \%$ del cortante basal \\
$\boldsymbol{F}$ & según Ec. 4 & 0.5 & $\mathrm{~F}_{\mathrm{t}}=10 \%$ del cortante basal \\
$\boldsymbol{G}$ & según Ec. 5 & 0.5 & $\mathrm{~F}_{\mathrm{t}}=10 \%$ del cortante basal \\
\hline
\end{tabular}

Las Ecs. 4 y 5 referidas en la Tabla 2 para el cálculo del factor de amplificación $\alpha$ son las siguientes (ver también Ec. 1a):

$\alpha=\left[2.25-2.0\left(e_{s} / b\right)\right] \geq 1.25$
$\alpha=\left[2.0-1.5\left(e_{s} / b\right)\right] \geq 1.25$

Para todos los casos el cortante basal en cada dirección se obtiene con ayuda de espectros de diseño del tipo Newmark-Hall correspondientes a un amortiguamiento del 5\% y a una probabilidad de excedencia de 50\%. En la dirección Y (Fig. 1), la aceleración máxima del terreno se supone igual a 1g, mientras que en la dirección $X$ se usan los valores de la Tabla 3 que resultan de escalar ambos componentes con el mismo factor de cada sismo. Los registros se escalaron mediante una simple factorización de las amplitudes de aceleración. En todos los casos se considera un factor de reducción de fuerzas sísmicas $\mathrm{R}=4$. Este valor se seleccionó simplemente para que la respuesta de los elementos resistentes estuviera en el intervalo no lineal. Este mismo valor fue usado también por Chandler y Duan [2]. El diseño considera el componente transversal del movimiento del terreno mediante un espectro de diseño similar al usado en dirección Y pero asociado a la aceleración máxima del terreno en dirección X para cada sismo. 
Tabla 3. Aceleraciones máximas del terreno

\begin{tabular}{|c|c|c|c|c|c|}
\hline \multirow[t]{2}{*}{ Registro } & \multicolumn{2}{|l|}{ original } & \multirow{2}{*}{$\begin{array}{l}\text { factor de } \\
\text { escala }\end{array}$} & \multicolumn{2}{|l|}{ escalado } \\
\hline & dirección $Y$ & dirección $X$ & & dirección $Y$ & dirección $X$ \\
\hline $\begin{array}{l}\text { Imperial Valley, El Centro, } \\
1940\end{array}$ & $0.35 \mathrm{~g}$ & $0.21 \mathrm{~g}$ & 2.871 & $1.00 \mathrm{~g}$ & $0.60 \mathrm{~g}$ \\
\hline Loma Prieta, Corralitos, 1989 & $0.63 \mathrm{~g}$ & $0.48 \mathrm{~g}$ & 1.588 & $1.00 \mathrm{~g}$ & $0.76 \mathrm{~g}$ \\
\hline $\begin{array}{l}\text { Michoacán, México, } \\
\text { La Unión, } 1985\end{array}$ & $0.17 \mathrm{~g}$ & $0.15 \mathrm{~g}$ & 5.81 & $1.00 \mathrm{~g}$ & $0.89 \mathrm{~g}$ \\
\hline
\end{tabular}

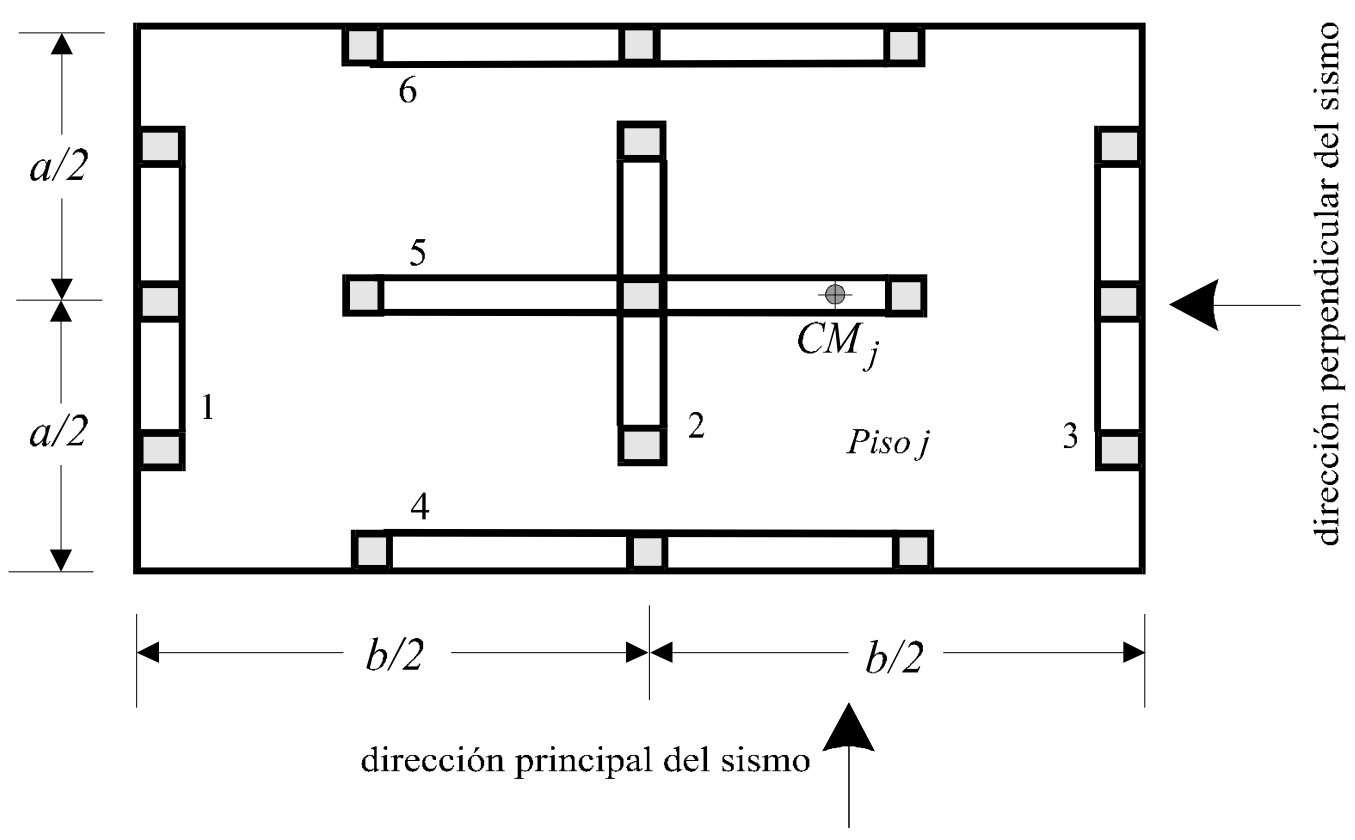

Figura 1. Planta del modelo considerado. 


\section{ANÁLISIS}

Definidos los valores de las fuerzas de fluencia de cada uno de los elementos resistentes de acuerdo con los diferentes procedimientos estáticos de la sección anterior, los modelos se someten a los tres registros sísmicos indicados en la Tabla 3 escalados para que la aceleración máxima del terreno en dirección $\mathrm{Y}$ sea de $1 \mathrm{~g}$ y de esta manera el diseño sea congruente con los espectros de diseño.

El análisis se lleva a cabo utilizando el método de aceleración constante y el método de NewtonRaphson modificado [13]. El parámetro que se reporta en este trabajo es la demanda de ductilidad de desplazamiento lateral de los elementos resistentes 1, 3 y 4 de cada uno de los entrepisos. En la modelación se ignoran las deformaciones axiales de los elementos resistentes a carga lateral, por lo que el estudio se limita a edificios de cortante. Para los modelos 5, 6 y 7 (Fig. 2) el elemento 1 corresponde al lado rígido y el elemento 3 al lado flexible del sistema.
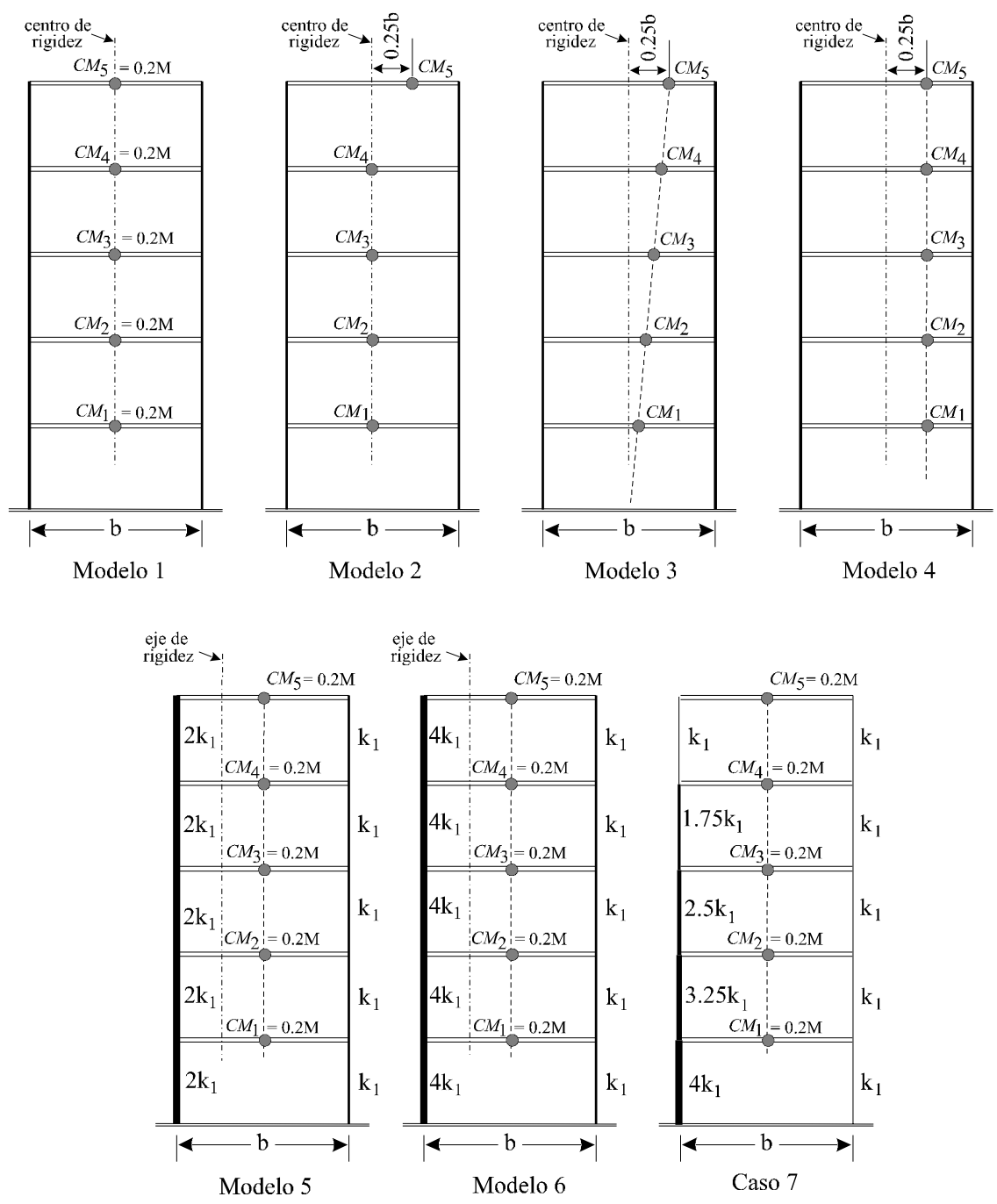

Figura 2. Elevaciones de los siete modelos estudiados. 


\section{RESULTADOS}

Las demandas de ductilidad correspondientes al procedimiento de diseño $\boldsymbol{a}\left(\alpha=1.0, \delta=0.0\right.$ y $\mathrm{F}_{\mathrm{t}}$ según UBC) se muestran en la Fig. 3 para los tres sismos considerados. En el lado izquierdo de la figura se presentan los resultados calculados para el elemento 1, en la parte central los del elemento 3 y en el lado derecho aquellos obtenidos para el elemento 4 (elemento transversal). En todos los casos el eje vertical identifica el entrepiso mientras que en las abscisas se lee la demanda calculada. Cada tipo de línea corresponde a uno de los siete modelos mostrados en la Fig. 2. El modelo de referencia (Modelo 1), correspondiente al sistema estructural sin desbalance torsional, se identifica con línea continua y círculos blancos. Como es de esperarse para este caso, las demandas de ductilidad para los elementos 1 y 3 resultan iguales entre si.
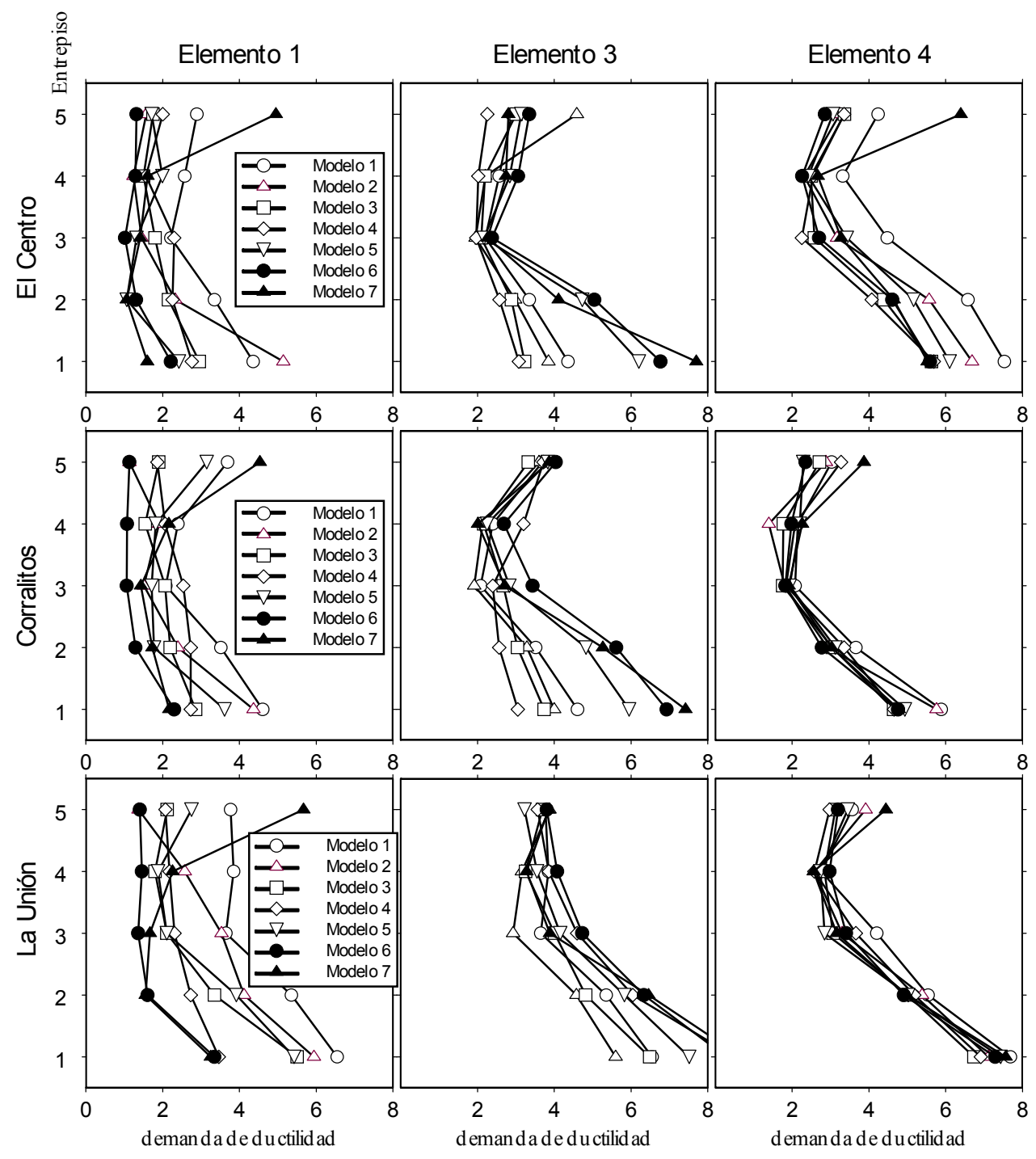

Figura 3. Demandas de ductilidad de sistemas diseñados con el procedimiento $a$. 
Por lo que se refiere al elemento 1, en las gráficas del lado izquierdo de la Fig. 3 (procedimiento de diseño a) se puede ver que, salvo por las demandas del entrepiso superior del modelo 7 y el entrepiso inferior del modelo 5, la demanda de ductilidad calculada para los modelos 2 a 7 es menor que la obtenida para el Modelo 1. Es importante observar que usualmente estos elementos se ven favorecidos por la torsión, por lo que el uso de $\delta=0.0$ en este procedimiento de diseño (a) es conservador ya que se traduce en no descontar en el diseño el cortante por torsión que les favorece.

Las tres gráficas centrales de la misma figura, las cuales corresponden al elemento 3, muestran que las demandas de ductilidad de los modelos torsionalmente desbalanceados 5, 6 y 7 exceden las demandas del modelo balanceado, principalmente en los entrepisos inferiores. El hecho de obtener demandas de ductilidad mayores que las del modelo balanceado para este elemento es razonable ya que el procedimiento de diseño $\boldsymbol{a}$ no amplifica la excentricidad de diseño al considerar $\alpha=1.0$. Es interesante observar que los modelos para los cuales la demanda excede la del caso balanceado (modelos 5, 6 y 7) son aquellos con excentricidad ocasionada por asimetría de rigidez.

Las gráficas derechas de la misma Fig. 3 muestran las demandas de ductilidad del elemento transversal 4. Se observa que, en general, la demanda de ductilidad de los modelos desbalanceados es prácticamente igual a la del modelo de referencia balanceado (Modelo 1). La única excepción importante se observa para el modelo 7 en donde la demanda es mayor que la del modelo de referencia en el entrepiso superior (esto se observa para los tres sismos).

Los resultados correspondientes al procedimiento de diseño $\boldsymbol{b}\left(\alpha=1.5, \delta=0.0\right.$ y $\mathrm{F}_{\mathrm{t}}$ según UBC) se muestran en la Fig. 4. Se puede observar que el uso de $\alpha=1.5$ no modifica apreciablemente las demandas de ductilidad del elemento 1 respecto a las obtenidas con el procedimiento de diseño $\boldsymbol{a}$ el cual emplea $\alpha=1.0$ (Fig. 3). Por otro lado, el uso de $\alpha=1.5$ sí reduce significativamente las demandas de los elementos del lado derecho (elemento 3) de los entrepisos inferiores, aun cuando para el elemento del entrepiso superior del modelo 7 las demandas aun son mayores que las calculadas para el caso torsionalmente balanceado (TB). Las demandas de ductilidad de los elementos transversales (elemento 4) calculadas para el procedimiento $\boldsymbol{a}$ prácticamente resultan iguales a las obtenidas al aplicar el procedimiento $\boldsymbol{b}$.

Las demandas de ductilidad calculadas para modelos diseñados con el procedimiento $c(\alpha=$ $1.0, \delta=0.5$ y $F_{t}$ según UBC) se muestran en la Figura 5. Este diseño es similar al $\boldsymbol{a}$ (Fig. 3) pero permite una reducción del $50 \%$ de las fuerzas cortantes ocasionadas por torsión para el diseño de los elementos del lado izquierdo (elemento 1). Las gráficas del lado izquierdo muestran que las demandas de ductilidad del elemento 1 crecen un poco en los entrepisos inferiores respecto a las calculadas con el procedimiento $\boldsymbol{a}(\delta=0.0)$, pero no exceden significativamente las demandas del caso TB. Esto sugiere que el uso de $\delta=0.5$ es razonable. La comparación de las gráficas centrales de las Figuras 3 y 5 (casos $\boldsymbol{a}$ y $\boldsymbol{c}$ ) muestra que el uso de $\alpha=1.0$ en combinación con $\delta=0.5$ no modifica apreciablemente las demandas de ductilidad de los elementos del lado derecho (éstas son prácticamente iguales a las mostradas en la Fig. 3). Por lo que se refiere a las demandas de los elementos transversales, las calculadas con el procedimiento $\boldsymbol{a}$ resultaron muy similares a las calculadas con el procedimiento $\boldsymbol{c}$. 


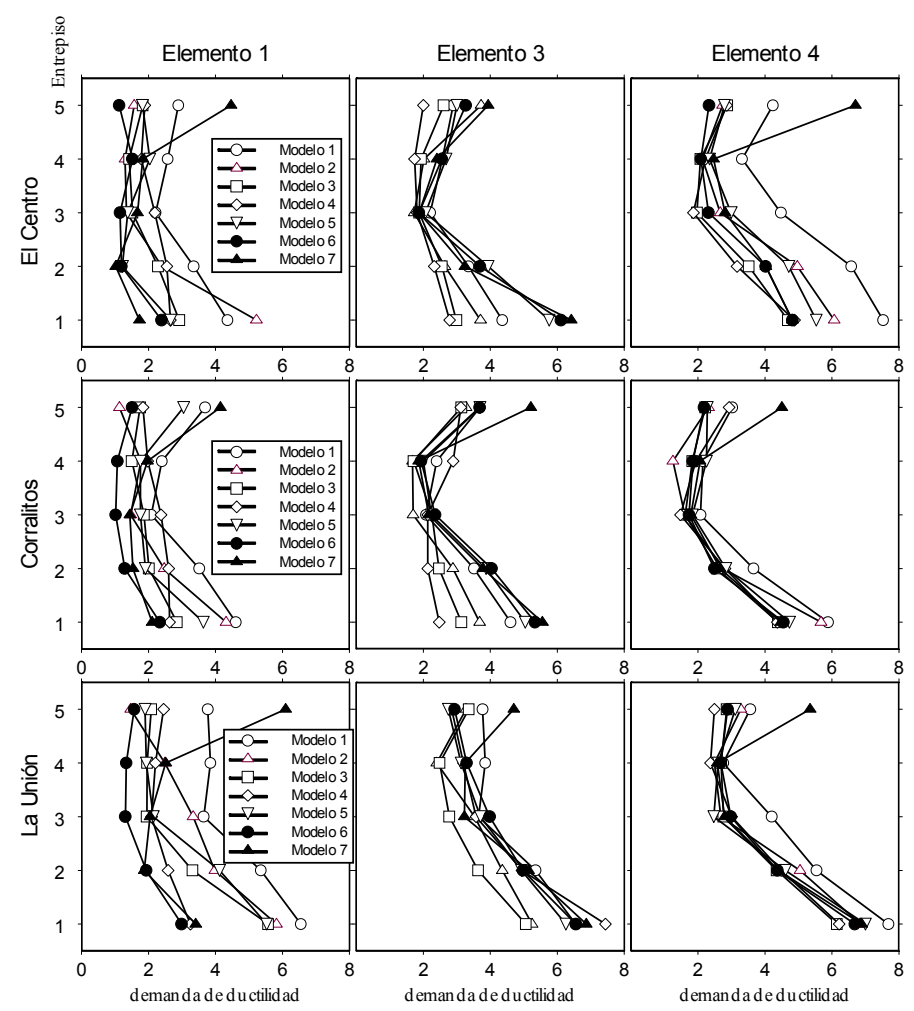

Figura 4. Demandas de ductilidad de sistemas diseñados con el procedimiento $b$.
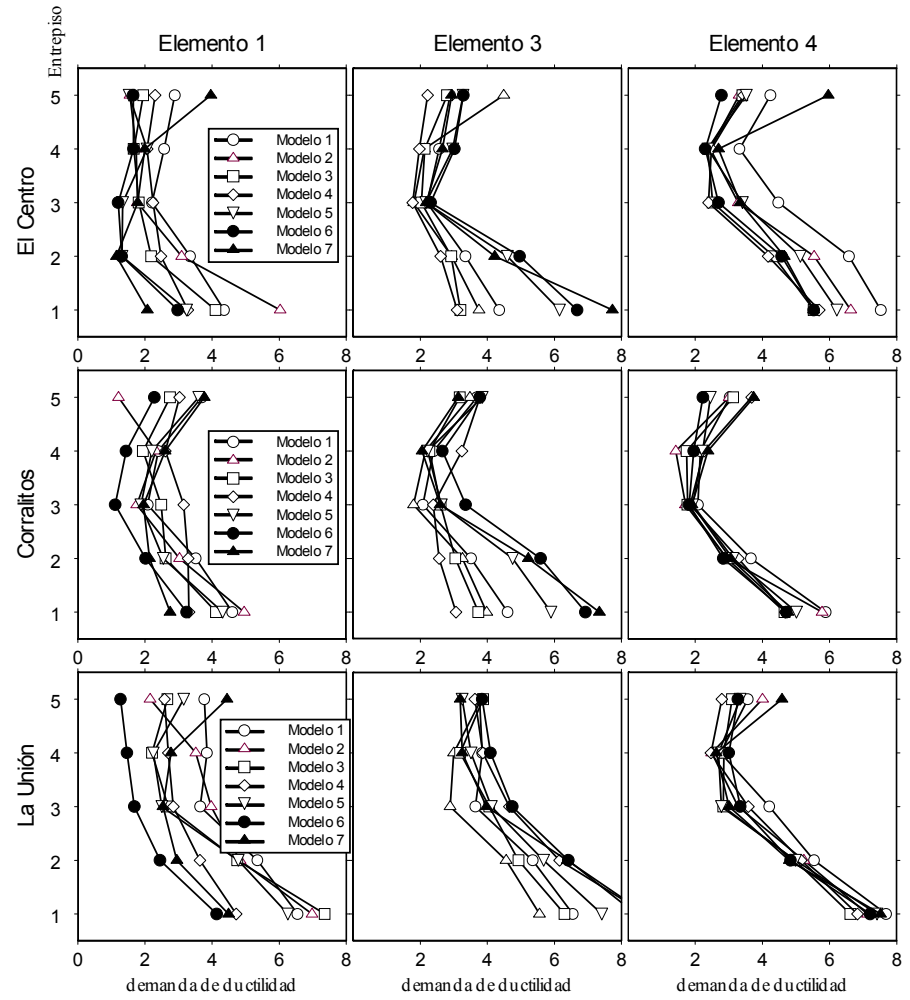

Figura 5. Demandas de ductilidad de sistemas diseñados con el procedimiento $c$. 
En la Figura 6 se muestran las demandas de ductilidad calculadas para elementos diseñados con el procedimiento $\boldsymbol{d}$ el cual usa $\alpha=1.5$ y $\delta=0.5$. Las gráficas del lado izquierdo muestran que las demandas de ductilidad para los elementos 1 de los modelos TD resultan menores que las correspondientes demandas de modelos $\mathrm{TB}$, en casi todos los casos. Por lo que se refiere a los elementos 3 , se observa que esta combinación de parámetros de diseño aplicados simultáneamente $(\alpha=$ $1.5 \mathrm{y} \delta=0.5$ ) conduce a valores de demandas de ductilidad de los modelos TD no mayores que $50 \%$ de los valores correspondientes del caso TB (Modelo 1). Además, comparando las gráficas centrales de las Figuras 4 y 6 , se observa que las demandas de ductilidad de estos elementos para $\alpha=1.5$ son prácticamente independientes de la selección de $\delta(0.0$ o 0.5$)$; excepto para entrepisos superiores donde se aprecia una ligera reducción con $\delta=0.5$. Similarmente, observando las gráficas izquierdas de las Figs. 5 y 6 se ve que el uso de $\delta=0.5$ conduce a las mismas demandas del elemento 1 , independientemente de si el diseño se combina $\operatorname{con} \alpha=1.0$ o con $\alpha=1.5$.

Las recomendaciones de Chandler y Duan [2], en el sentido de emplear un valor de $\alpha$ variable (en función de la excentricidad), $\delta=0.5$ y $_{\mathrm{t}}=10 \%$ del cortante basal, se analizan con la Fig. 7. Es importante destacar que no se incluye el factor de resistencia a aplicar al elemento 1 que también se sugiere en la misma referencia 2. Las comparaciones de estas recomendaciones de diseño $(\boldsymbol{e})$ se hacen con respecto a las del caso $\boldsymbol{d}\left(\alpha=1.5, \delta=0.5\right.$ y $\mathrm{F}_{\mathrm{t}}$ según UBC) mostradas en la Fig. 6 ya que este procedimiento $(\boldsymbol{d})$ conduce a demandas de ductilidad de los elementos 1, 3 y 4 de los modelos TD más próximas a las demandas del modelo TB que los procedimientos $\boldsymbol{a}, \boldsymbol{b}$ o $\boldsymbol{c}$. En el lado izquierdo de la Fig. 7 se observa que las demandas de ductilidad del elemento 1 son prácticamente iguales a las obtenidas al usar el procedimiento $\boldsymbol{d}$ de la Fig. 6, excepto para los pisos superiores donde el procedimiento basado en las recomendaciones de Chandler y Duan conduce a menores demandas de ductilidad. Por lo que se refiere a las demandas de ductilidad calculadas para los elementos 3 y 4 se observa que este procedimiento de diseño $\boldsymbol{e}$ conduce a demandas de ductilidad similares a las calculadas usando el procedimiento $\boldsymbol{d}$ pero también con reducciones para entrepisos superiores. Esto se debe al uso de la fuerza $F_{t}=10 \%$ del cortante basal independientemente del valor del periodo de vibración de la estructura. En este caso por ser $\mathrm{T}=0.5 \mathrm{~s}$ el procedimiento de diseño $\boldsymbol{d}$, el cual se basa en el UBC para determinar $F_{t}$, conduce a $F_{t}=0.0$.

Los procedimientos de diseño $\boldsymbol{f}$ y $\boldsymbol{g}$, los cuales son muy similares al $\boldsymbol{e}$ pero con fórmulas más sencillas para el cálculo de $\alpha$, conducen a las demandas de ductilidad mostradas en las Figs. 8 y 9. Los resultados son prácticamente iguales a los comentados para el procedimiento $\boldsymbol{e}$.

\section{Conclusiones}

En general, los procedimientos de diseño considerados en este trabajo con $\delta=0.0$ conducen a demandas de ductilidad del elemento 1 de sistemas torsionalmente desbalanceados (TD) menores que las calculadas para el mismo elemento pero de sistemas torsionalmente balanceados (TB).

El uso de $\alpha=1.0$ conduce a demandas de ductilidad de los elementos 3 (derechos) de sistemas torsionalmente desbalanceados (TD) mayores que las correspondientes de sistemas TB, principalmente para entrepisos inferiores. 


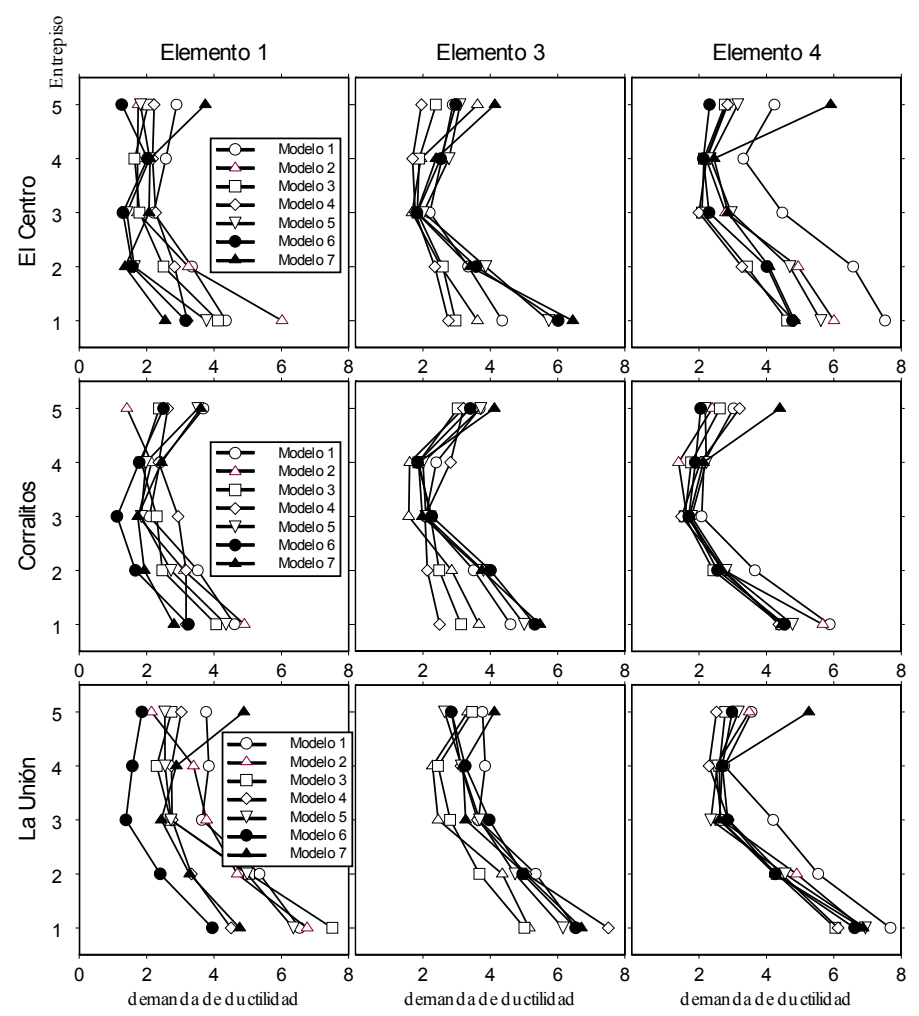

Figura 6. Demandas de ductilidad de sistemas diseñados con el procedimiento $d$.

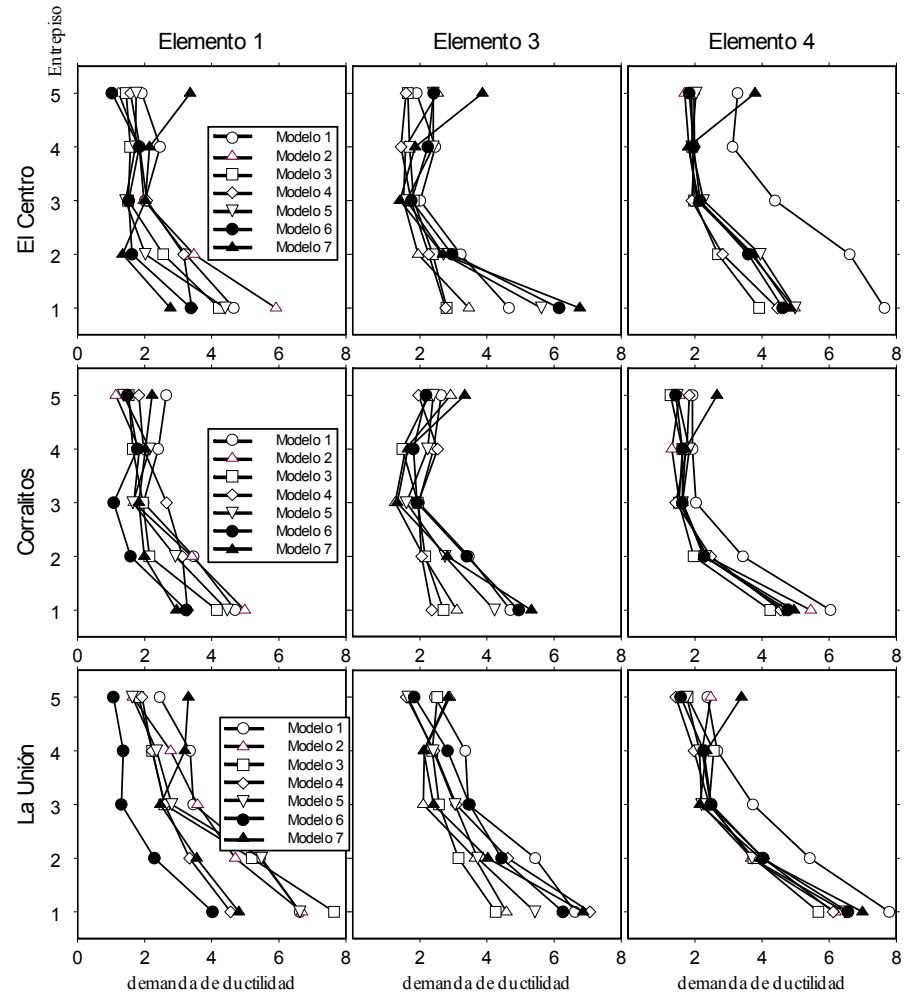

Figura 7. Demandas de ductilidad de sistemas diseñados con el procedimiento $e$. 

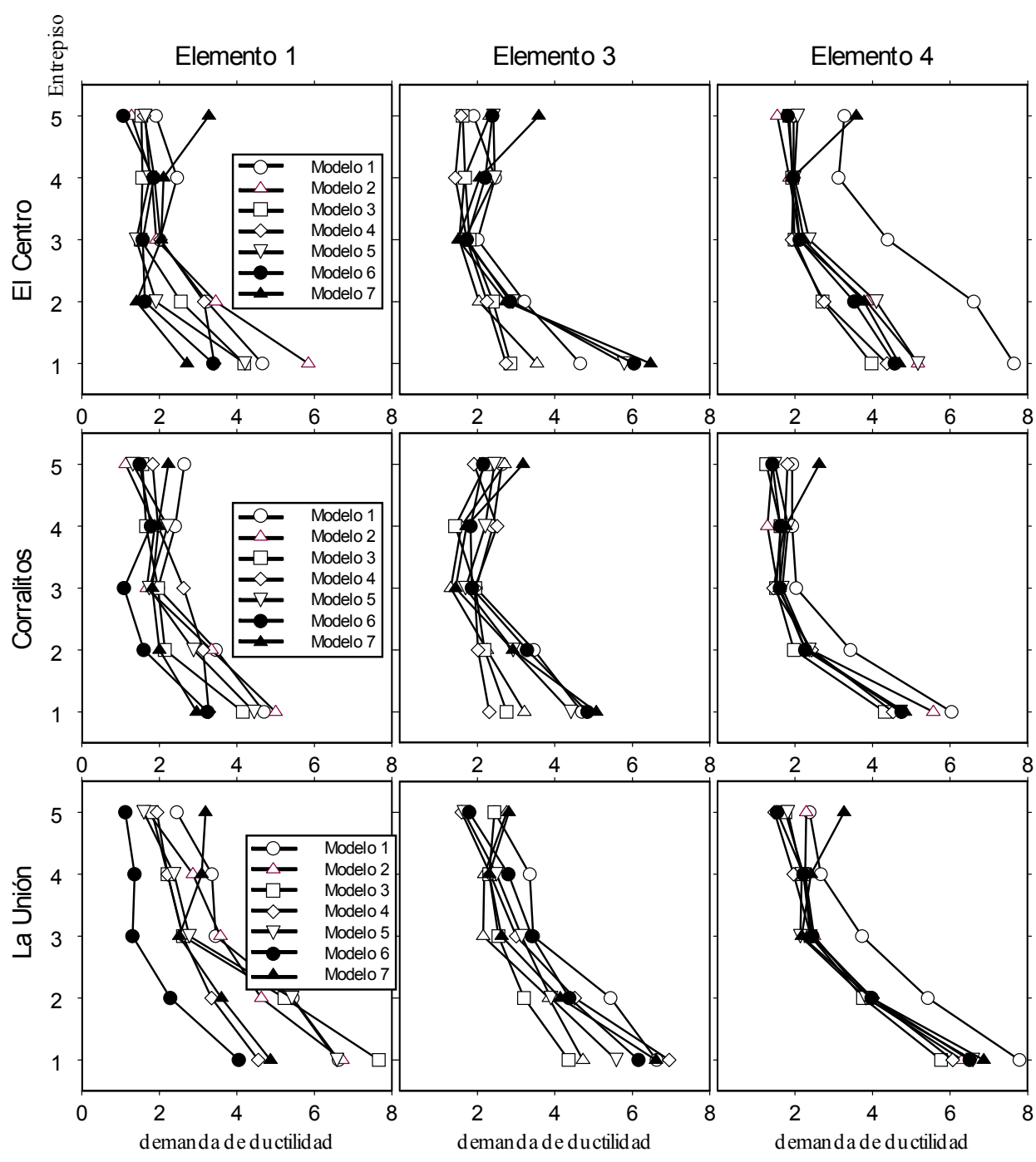

Figura 8. Demandas de ductilidad de sistemas diseñados con el procedimiento $f$.

El uso de $\alpha=1.0$ o $\alpha=1.5$ prácticamente conduce a las mismas demandas de ductilidad del elemento 1 (elemento izquierdo). El uso de $\alpha=1.5$ reduce significativamente las demandas de ductilidad de los elementos del lado derecho. Por ello, se recomienda usar $\alpha=1.5$.

La combinación de coeficientes $\alpha=1.5$ y $\delta=0.5$ conduce a valores de demandas de ductilidad para los elementos considerados de los modelos TD muy cercanos o menores a las correspondientes de los modelos TB, por lo que se recomienda su uso en el caso de emplear el método estático de diseño por torsión.

Para los elementos 1, 3 y 4, las recomendaciones de Chandler y Duan consideradas en este trabajo prácticamente conducen a las mismas demandas de ductilidad calculadas con el procedimiento de diseño que usa $\alpha=1.5, \delta=0.5 \mathrm{y} \mathrm{F}_{\mathrm{t}}$ del UBC (procedimiento de diseño $\boldsymbol{d}$ ), excepto para pisos superiores donde las recomendaciones de Chandler y Duan conducen a demandas un poco menores. 
Por ello, se sugiere emplear $\mathrm{F}_{\mathrm{t}}=10 \%$ del cortante basal y sin incluir el factor de resistencia que proponen Chandler y Duan para incrementar la capacidad del elemento del lado rígido. Tampoco se considera necesario ubicar los centros de resistencia referidos por Duan y Chandler [6].

Las fórmulas para el cálculo del coeficiente $\alpha$ de los procedimientos $\boldsymbol{f}$ y $\boldsymbol{g}$ prácticamente conducen a las mismas demandas de ductilidad que el procedimiento que incluye las recomendaciones de Chandler y Duan consideradas en este estudio. Por ello, se recomiendan los procedimientos de diseño $\boldsymbol{d}, \boldsymbol{e}$ y $\boldsymbol{f}$.
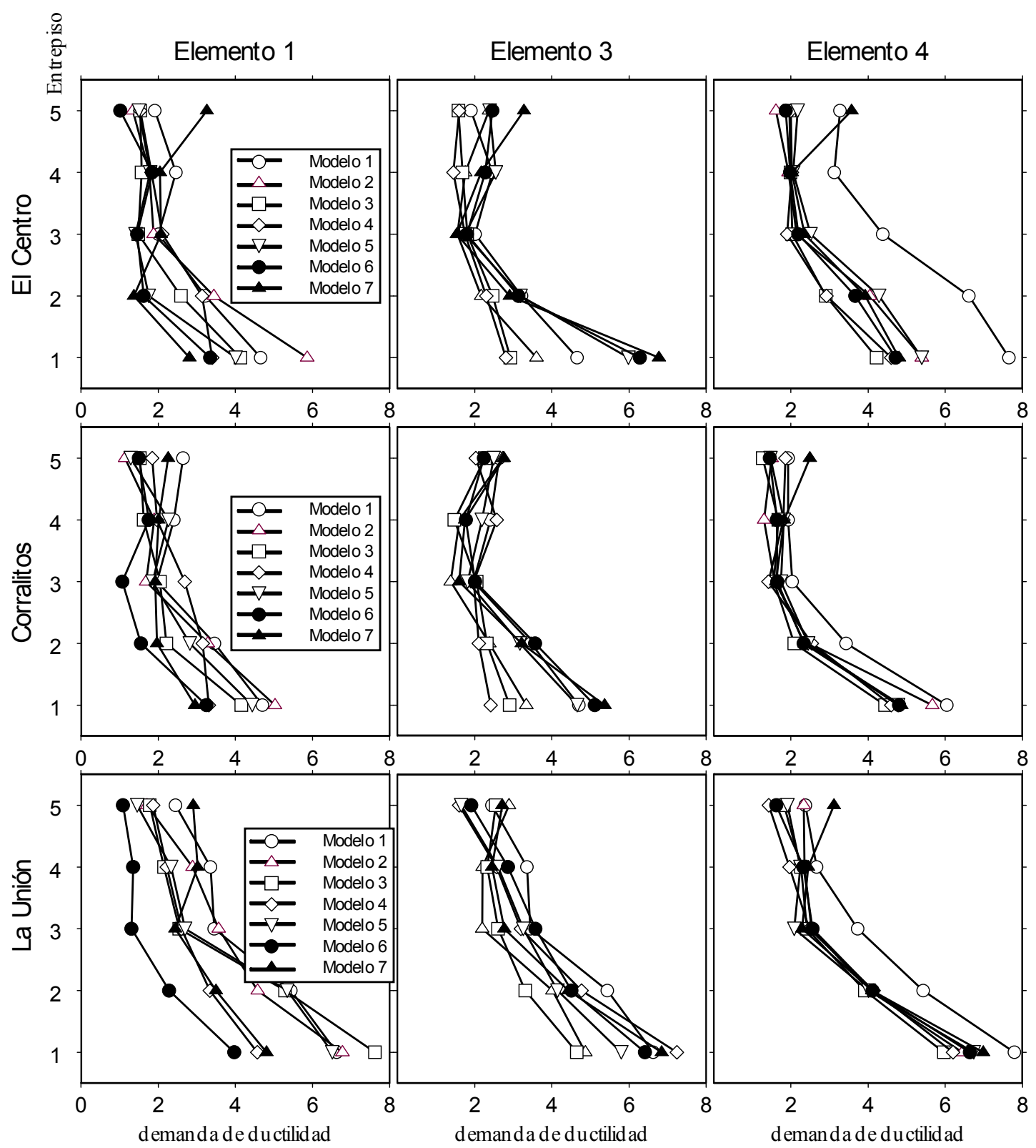

Figura 9. Demandas de ductilidad de sistemas diseñados con el procedimiento $g$. 


\section{REFERENCIAS}

1. Duan, X. N. y Chandler, A. M. (1993). 'Inelastic seismic response of code-designed multistory frame buildings with regular asymmetry'. Earthquake Engineering and Structural Dynamics, 22(5), 431-445.

2. Chandler, A. M. y Duan, X. N. (1993). 'A modified static procedure for the design of torsionally unbalanced multistorey frame buildings'. Earthquake Engineering and Structural Dynamics, 22(5), 447-462.

3. Páez, A. y Escobar, J. A. (2000) 'Diseño de edificios por torsión, requisito complementario'. El Portulano de la Ciencia, Instituto Politécnico Nacional, 1(3), 111-126.

4. Tso, W. K. y Wong, C. M. (1993) 'An evaluation of the New Zealand code torsional provision'. Bulletin of the New Zealand National Society for Earthquake Engineering, 26(2), 194-207.

5. De la Llera, J. C. y Chopra, A. K., (1994) 'Accidental and natural torsion in earthquake response and design of buildings', Report No. EERC 94-07, University of California, Berkeley, CA.

6. Duan, X. N. y Chandler, A. M. (1995). 'Seismic torsional response and design procedures for a class of setback frame buildings'. Earthquake Engineering and Structural Dynamics, 24, 761777.

7. Correnza, J. C. y Hutchinson, G. L. (1994) 'Effect of transverse load-resisting elements on inelastic earthquake response of eccentric-plan buildings', Earthquake Engineering and Structural Dynamics, 23, 75-89.

8. Humar, J. L. y Kumar, P. (1999) 'Effect of orthogonal inplane structural elements on inelastic torsional response' Earthquake Engineering and Structural Dynamics, 28, 1071-1097.

9. International Conference of Building Officials (1994), Uniform Building Code; 1994 Edition, Whittier, California.

10. Tso, W. K. (1990) 'Static eccentricity concept for torsional moment estimation,' Journal of Structural Engineering, 116(5), 1199-1212.

11. Normas Técnicas Complementarias para Diseño por Sismo (1995). Gaceta Oficial del Distrito Federal, México.

12. Wong, C. M. y Tso, W. K. 'Evaluation of seismic torsional provisions in Uniform Building Code,' Journal of Structural Engineering, 121(10), 1436-1442.

13. Chopra, A. K. (1995). Dynamics of Structures - Theory and Applications to Earthquake Engineering. Prentice Hall. 